\title{
Law for the Construction Industry
}


Other Macmillan titles in Building and Surveying

Administrative Law for the Construction Industry J. R. Lewis

Introduction to Urban and Land Economics

P. N. Balchin and J. L. Kieve

Building Technology Ivor H. Seeley

Building Maintenance Ivor H. Seeley

Building Quantities Explained Ivor H. Seeley

Advanced Building Quantities Ivor H. Seeley and R. Winfield

Computers in Quantity Surveying R. J. Alvey

Quantitative Methods in Construction Management and Design

J. F. Woodward 


\title{
LAW FOR THE CONSTRUCTION INDUSTRY
}

\author{
J. R. LEWIS, LL.B., A.C.I.S. \\ Barrister at Law
}




\title{
(C) J. R. Lewis 1976
}

Softcover reprint of the hardcover 1st edition 1976

All rights reserved. No part of this publication may be reproduced or transmitted, in any form or by any means, without permission.

\author{
First published 1976 by \\ THE MACMILLAN PRESS LTD \\ London and Basingstoke \\ Associated companies in New York Dublin \\ Melbourne Johannesburg and Madras
}

SBN 333190378 (hard cover)

SBN 333190386 (paper cover)

ISBN 978-0-333-19038-8 ISBN 978-1-349-15713-6 (eBook)
DOI 10.1007/978-1-349-15713-6

Produced by computer-controlled phototypesetting, using OCR input techniques, and printed offset by UNWIN BROTHERS LIMITED

The Gresham Press, Old Woking, Surrey

This book is sold subject to the standard conditions of the Net Book Agreement.

The paperback edition of this book is sold subject to the condition that it shall not, by way of trade or otherwise, be lent, resold, hired out, or otherwise circulated without the publisher's prior consent, in any form of binding or cover other than that in which it is published and without a similar condition including this condition being imposed on the subsequent purchaser. 


\section{Contents}

Preface vii

Table of Cases viii

Table of Statutes xiii

1 The English Legal System 1

2 Law and Government 12

3 Liability under the Law 21

4 The Law of Contract $\quad 35$

5 Special Contracts $\quad 69$

6 Liabilities in Tort $\quad 93$

7 Rights in Property 120

8 Landlord and Tenant 137

\section{Appendixes}

1 Statutory Provisions relating to Residential, Business and Agricultural Tenancies $\quad 165$

2 Standard Form of Building Contract 169

3 Case References 176

$\begin{array}{ll}\text { Index } & 207\end{array}$ 


\section{Preface}

This book first made its appearance under the title Building Law. Developments in the law, changes in the construction industry, and the emergence of new syllabuses for the examinations of the Royal Institution of Chartered Surveyors have made changes in format and title of this book necessary.

An expansion of the text has meant that the material would be too lengthy for one volume so two volumes are now necessary. This particular book, dealing with the general principles of law applying to the construction industry, is designed to cover the Part I introductory examination and the Part II Law of Property examination in the General Practice syllabuses of the RICS. Part I (Elements of Law) and Part II (Law of Contract) syllabuses for the Quantity Surveying Section of the RICS are also covered.

The book does not pretend to be an exhaustive statement of the law; rather it covers the legal matters dealt with in these syllabuses and which touch upon the work of the building contractor, the surveyor and the architect. As far as is possible, legal terminology has been dispensed with, footnotes discarded, and an emphasis placed upon simplicity and readability.

October 1975

J. R. L. 


\section{Table of Cases}

Aldin v. Latimer Clarke, [1894] 2 Ch. $437 \quad 148$

Alphacell Ltd v. Woodward, [1972] A.C. 824 122, 180

Amalgamated Building Contractors Ltd v. Waltham Holy Cross

U.D.C., [1952] 2 All E.R. 452 63, 180

Andreae v. Selfridge \& Co. Ltd, [1938] Ch. 1 109, 110, 180

Angell v. Jay, [1911] 1 K.B. 666 66, 180

Arcos Ltd v. Ronaasen \& Son Ltd, [1933] A.C. 470 52, 180

Ashbury Railway Co. v. Riche (1875), L.R. 7 H.L. 653 27, 180

Attwood v. Small (1838), 6 Ch. \& F. 232 49, 181

Bailey v. De Crespigny (1869), L.R. 4 Q.B. 180 60, 181

Baker v. Hopkins, [1959] 1 W.L.R. 966101

Balfour v. Barty-King, [1957] 1 Q.B. 49696,181

Barrington v. Lee, The Times, 29 Oct. 1971 90, 181

Bell v. Lever Bros Ltd, [1932] A.C. 161 81, 181

Bower v. Peate (1876), l Q.B.D. $321 \quad 110,182$

Bradford Corporation v. Pickles, [1895] A.C. 587 94, 122, 182

Breams Property Trust v. Stroulger, [1948] 2 K.B. 1 162, 182

Brogden v. Metropolitan Railway (1877), 2 App. Cas. 666 37, 182

Bufe v. Turner (1815), 6 Taunt 338 50, 182

Burt v. Cousins \& Co. Ltd, [1971] 2 Q.B. 426 90, 182

Byrne v. Van Tienhoven (1880) 5 C.P.D. 344 39, 183

Cavanagh v. Ulster Weaving Co. Ltd, [1959] 2 All E.R. 745 99, 183

Central London Property Ltd v. High Trees House Ltd, [1947] K.B. $13032,41,152,183$

Century Insurance Co. Ltd v. Northern Ireland Road Transport Board, [1942] A.C. 509 97, 183

Chambers v. Goldthorpe, [1901] 1 K.B. 624 87, 183

Christie Owen \& Davies Ltd v. Rapacioli, [1974] 2 W.L.R. 723 91, 184

Christmas v. General Cleaning Contractors Ltd, [1953] A.C. 180105

Clark v. Woor, [1965] 1 W.L.R. 650 67, 184

Clay v. A. J. Crump Ltd, [1964] 1 Q.B. 533 86, 107, 184

Clayton v. Woodman Ltd, [1962] 2 All E.R. 33 86, 107, 185

Conway v. George Wimpey \& Co. Ltd, [1951] 1 All E.R. 56 95, 185 
Cowern v. Nield, [1912] 2 K.B. 419 42, 185

Crane v. Morris, [1965] 1 W.L.R. 1104144

Cranleigh Precision Engineering Ltd v. Bryant, [1964] 3 All E.R. 289 81,185

Craven Ellis v. Canons Ltd, [1936] 2 K.B. 403 67, 185

Cricklewood Property, etc., Trust Ltd v. Leighton's Investment Trust Ltd, [1945] A.C. 221 60, 152, 186

Croft v. William F. Blay Ltd, [1919] 2 Ch. 343 162, 186

Croshaw v. Pritchard (1899), 16 T.L.R. 45 36, 186

Cummings v. Arrol \& Co. Ltd, [1962] 1 W.L.R. 295 82, 186

Cunliffe v. Hampton Wick Local Board (1893), H.B.C., 4th edn, ii p. $370 \quad 58,186$

D \& C Builders v. Rees, [1966] 2 Q.B. 617 41, 187

Dakin \& Co Ltd v. Lee, [1916] 1 K.B. 566 53, 187

Dalton v. Angus \& Co. (1881), 6 App. Cas. 740 129, 187

Davis Contractors Ltd v. Fareham U.D.C., [1956] A.C. 696 59, 60, 187

Denny v. Supplies \& Transport Co. Ltd, [1950] 2 K.B. 374 97, 187

Denny, Mott \& Dickson Ltd v. Fraser \& Co. Ltd, [1944] A.C. 265 60, 187

Dewar v. Mintoft, [1912] 2 K.B. 373 70, 188

Donoghue v. Stevenson, [1932] A.C. 362103

Doughty v. Turner Manufacturing Co., [1964] 1 Q.B. 518 100, 188

East Ham U.D.C. v. Bernard Sunley \& Sons Ltd, [1965] 3 All E.R. 61958,188

Electrochrome Ltd v. Walsh Plastics Ltd, [1968] 2 All E.R. $205 \quad$ 100, 188 Ellis v. Sheffield Gas Consumers Council (1853), 2 E. \& B. 767 96, 188

Fay v. Miller, Wilkins \& Company, [1941] Ch. 360 90, 188

Feather v. Keighley Corporation (1953), 52 L.G.R. 30 57, 189

Felton v. Wharrie (1906), H.B.C., 4th edn, ii, p. 398 57, 189

G.N.R. v. Witham (1873), L.R. 9 C.P. $16 \quad 37,189$

Gallagher v. Humphrey (1862), 6 L.T. (N.s.) $684 \quad 105,189$

Gloucestershire C.C. v. Richardson, [1969] 1 A.C. 480 92, 189

Greaves Contractors Ltd v. Baynham Meikle, The Times, 16 May 1975 82, 205

Gregory v. Piper (1829), 9 B. and C. $591 \quad 112,189$

Hadley v. Baxendale (1854), 9 Excheq. $341 \quad 62,189$

Hanson v. Newman, [1934] Ch. 298157

Harbutt's Plasticine v. Wayne Tank and Pump Co., [1970] 1 Q.B. 44761,190

Harris v. James (1876), 45 L.J.Q.B. $545 \quad 110,190$

Harrison v. Wells, [1967] 1 Q.B. 263162 
Haseldine v. Daw \& Son Ltd, [1941] 2 K.B. 343 105, 190

Heaven v. Pender (1883), 11 Q.B.D. 503 103, 107, 190

Henshaw v. Rochdale Corporation, [1944] K.B. 381 58, 190

Hickman \& Co. v. Roberts, [1913] A.C. 229 55, 84, 86, 190

Hinton v. Sparkes (1868), L.R. 3 C.P. 161158

Hoare v. Macalpine, [1932] 1 Ch. 167 113, 191

Hoenig v. Isaacs, [1952] 2 All E.R. 176 52, 53, 57, 62, 191

Holliday v. National Telephone Co., [1899] 2 Q.B. 392 96, 104, 191

Houlder Bros \& Co. v. Gibbs, [1925] Ch. 575160

Hounslow Borough Council v. Twickenham Garden Development

Ltd, [1971] Ch. 223 124, 191

Howatson v. Webb, [1907] 1 Ch. 537 47, 191

Hughes v. Percival (1883), 8 App. Cas. 443 96, 104, 191

Hussey v. Palmer, [1972] 1 W.L.R. 1286 41, 191

Ilford U.D.C. v. Beal, [1925] 1 K.B. 671 110, 192

Inwards v. Baker, [1965] 2 Q.B. 29 124, 192

Kelly v. Rogers, [1892] 1 Q.B. $910 \quad 150$

Konskier v. Goodman, [1928] 1 K.B. 421 112, 192

Langbrook Properties Ltd v. Surrey C.C., [1970] 1 W.L.R. $161 \quad$ 109, 192

Lavender v. Betts, [1942] 2 All E.R. 72150

Law v. Redditch Local Board, [1892] 1 Q.B. 127 63, 192

Leicester Board of Guardians v. Trollope (1911), 75 J.P. $197 \quad 86,192$

Limpus v. London General Omnibus Co. (1862), 1 H. \& C. 526 95, 192

Lloyd v. Grace, Smith \& Co., [1912] A.C. 716 29, 193

Long v. Lloyd [1958] 1 W.L.R. 753 66, 193

Long v. Millar (1879), 4 C.P.D. 450 71, 145, 193

Lurcott v. Wakeley, [1911] 1 K.B. 905154

Lynch v. Thorne, [1956] 1 W.L.R. 303 46, 193

McCutcheon v. David McBrayne Ltd, [1964] 1 W.L.R. 125 39, 194 Mackay v. Dick (1881), 6 App. Cas. 251 55, 193

Maddison v. Alderson (1883), 8 App. Cas. 467 71, 146, 193

Mainwaring v. Billington, [1952] 2 All E.R. 747 115, 194

Marsden U.D.C. v. Sharp (1931), 47 T.L.R. 549 58, 194

Meikle v. Maufe, [1941] 3 All E.R. 144134

Mersey Docks \& Harbour Board v. Coggins \& Griffith Ltd, [1947] A.C. 196,194

Mertens v. Home Freeholds Co., [1921] 2 K.B. 526 60, 62, 194

Metropolitan Water Board v. Dick, Kerr \& Co. Ltd, [1918] A.C. $119,59,60,194$

Midwood v. Manchester Corporation, [1905] 2 Q.B. 597 109, 195 
Notts Patent Brick \& Tile Co. v. Butler (1887), 16 Q.B.D. $778 \quad$ 50, 195

Padbury v. Holliday \& Greenwood Ltd (1912), 28 T.L.R. 494 96, 195

Panamena v. Leyland \& Co., [1947] A.C. 428 55, 195

Paris v. Stepney Borough Council, [1951] A.C. 367 106, 195

Parkinson \& Co. Ltd v. Commissioner of Works, [1949] 2 K.B. 632 67,195

Patman \& Fotheringham v. Pilditch (1904), H.B.C., 6th edn, ii, p. 36856,196

Pearce v. Gardner, [1897] 1 Q.B. 688 70, 145

Pearson Ltd v. Dublin Corporation, [1907] A.C. 351 48, 196

Phillimore v. Lane (1925), 133 L.T. 268153

Potter v. Loppert, [1973] 2 W.L.R. 469 90, 196

Priestley v. Stone (1888), 4 T.L.R. 730 48, 89, 196

Pwllbach Colliery Co. Ltd v. Woodman, [1915] A.C. 634 110, 196

R. v. Hereford Corporation, [1970] 1 W.L.R. 1424 30, 197

R. v. Hussey (1924), 18 C.R. App. R. 121 (C.C.A.) 164, 197

Ranger v. G.W.R. (1854), 5 H.L. Cas. 72 63, 197

Rawlinson v. Ames, [1925] Ch. 96 71, 146, 197

ReCasey's Patents, [1892] 1 Ch. 104 40, 197

ReMoore \& Co. and Landauer \& Co., [1921] 2 K.B. 519 75, 198

Rickards Ltd v. Oppenheim, [1950] 1 K.B. 616 77, 198

Roberts v. Leicestershire C.C., [1961] Ch. 555 66, 198

Robinson v. Kilvert (1889), 41 Ch.D. 88 109, 198

Rogers v. James (1891), 8 T.L.R. $67 \quad 87,198$

Rylands v. Fletcher (1868), L.R. 3 H.L. 330 94, 112, 113, 198

Sage v. Spiers \& Ponds Ltd (1915), 31 T.L.R. 204 39, 199

Salford Corporation v. Lever, [1891] 1 Q.B. $168 \quad 34,199$

Salomon v. Salomon \& Co., [1897] A.C. 22 26, 199

Saunders v. Anglia Building Society, [1971] A.C. 1039 47, 199

Scammell v. Ouston, [1941] A.C. 251 39, 199

Shanklin Pier Ltd v. Detel Products Ltd, [1951] 2 K.B. 854 49, 199

Shanly v. Ward (1913), 29 T.L.R. 714160

Sharpe v. E.T. Sweeting Ltd, [1963] 1 W.L.R. 665 103, 200

Shaw v. Anthony (1939), 133 Est. Gaz. 342156

Smith v. Baker \& Sons, [1891] A.C. 325101

Smith v. Land \& House Corporation, [1884] 28 Ch.D. 7 49, 200

Smith v. Leech, Brain \& Co. Ltd, [1962] 2 Q.B. 405200

Smith v. Scott, [1972] 3 All E.R. 645 110, 200

Smith v. Vange Scaffolding \& Engineering Co. Ltd, [1970] 1 W.L.R.

77392,200 
Southern Portland Cement v. Cooper, [1974] 2 W.L.R. 152 105, 202 Spicer Ltd v. Mansell, [1970] 1 W.L.R. 333 25, 201

Stansbie v. Troman, [1948] 2 Q.B. 48 100, 201

Stevens v. Gourley (1859), 7 C.B. (N.s.) 99 50, 201

Stevenson v. McLean (1880), 5 Q.B.D. 346 37, 201

Stilk v. Myrick (1809), 2 Camp. 317 40, 201

Sturges v. Bridgman (1879), 11 Ch.D. 852 109, 110, 201

Sumpter v. Hedges, [1898] 1 Q.B. 673 54, 67, 202

Sutcliffe v. Thackrah, [1973] 1 W.L.R. 888 87, 202

Taylor v. Caldwell (1883), 32 L.J.Q.B. 164 59, 202

Tickner v. Buzzacott, [1965] Ch. 426 163, 202

Tinn v. Hoffman \& Co. (1873), 29 L.T. 271 37, 202

Townsend Builders Ltd v. Cinema News Ltd, [1959] 1 W.L.R. 119 50, 202

Trollope \& Colls v. Atomic Power Constructions Ltd, [1963] 1 W.L.R.

333203

Tulk v. Moxhay (1848), 2 Ph. 774 118, 203

Upfill v. Wright, [1911] 1 K.B. 506152

Victoria Laundry Ltd v. Newman Industries Ltd, [1949] 2 K.B. 52862,203

Vigers v. Cooke, [1919] 2 Q.B. 475 54, 203

Von Hatzfield-Wildenburg v. Alexander, [1912] 1 Ch. 28470

Walton Harvey v. Walker \& Homfrays Ltd, [1913] 1 Ch. 274 60, 203

Whitworth v. Miller, [1970] A.C. 583 68, 204

William Lacey v. Davis (1957), H.B.C., 6th edn, ii, p. 372 36, 204

Williams v. Fitzmaurice (1858), 32 L.T. (o.s.) 149 56, 204

Wilson v. Love, [1896] 1 Q.B. 626158

Wolverhampton Corporation v. Emmons, [1901] 1 Q.B. 515 64, 204

Wragg v. Lovatt, [1948] W.N. 455 70, 204

Wyld v. Silver, [1962] 3 All E.R. 309 7, 127, 204

Young v. Smith (1879), H.B.C., 4th edn, ii, p. 70 89, 205 


\title{
Table of Statutes
}

\author{
Statute of Frauds $1677 \quad 42$ \\ Prescription Act 1832128 \\ Customs and Inland Revenue Duties Act 186930 \\ Judicature Act 1873 6, 7, 124 \\ Infants Relief Act $1874 \quad 42$ \\ Partnership Act 189024 \\ Sale of Goods Act 1893 72, 74, 75, 76, 77, 79 \\ Bankruptcy Act 1914 28, 162 \\ Registration of Business Names Act 191625 \\ Settled Land Act 1925 138, 139, 140 \\ Law of Property Act 1925 42, 69, 70, 71, 89, 121, 123, 124, 127, \\ $131,135,136,138,139,140,145,146,147,158,159,162,163$ \\ section 1 121, 124, 143

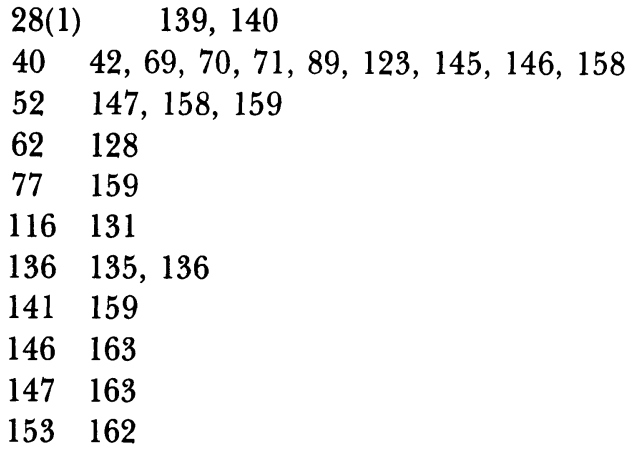 \\ Land Registration Act 1925 140, 141, 143, 158 \\ Land Charges Act 1925 141, 142, 143, 144 \\ Landlord and Tenant Act 1927 123, 157, 160, 163 \\ Public Health Act 1936 82, 122, 157 \\ Leasehold Property (Repairs) Act 1938 126, 163 \\ Limitation Act 1939 67, 153 \\ Law Reform (Contributory Negligence) Act 1945101 \\ Agricultural Holdings Act 1948 163, 167 \\ Companies Act $1948 \quad 43$ \\ Factories Act 1948 114, 119
}


$x i v$

Arbitration Act 195034

Landlord and Tenant Act 1954 123, 163, 164, 166, 167

Mines and Quarries Act $1954 \quad 114$

Restrictive Practices Act 19569

Rent Act $1957 \quad 165$

Occupiers Liability Act 1957 103, 104, 105, 119, 156

Housing Act 1957 155, 157, 164

Agriculture Act 1958167.

Tribunals and Enquiries Act 195819

Rights of Light Act 1959129

Corporate Bodies Contracts Act $1960 \quad 43$

Housing Act 1961 153, 154, 155, 157

London Government Act 1963155

Offices Shops and Railway Premises Act 1963 116, 117, 119

Water Resources Act 1963122

Limitation Act $1963 \quad 67$

Housing Act 1964 121, 149, 157

Protection from Eviction Act $1964 \quad 168$

Resale Prices Act 19649

Hire Purchase Act 196580

Race Relations Act 1965161

Misrepresentation Act $1967 \quad 48$

Rent Act 1968 150, 165, 166, 167, 168

Employers Liability (Defective Equipment) Act 1969 81, 104

Animals Act $1971 \quad 114$

Industrial Relations Act 1971 9, 31

Contract of Employment Act 197280

European Communities Act 197227

Defective Premises Act 1972103

Local Government Act 1972 17, 30

Housing Finance Act $1972 \quad 165$

Sale of Goods (Implied Terms) Act 1973 73, 74, 76, 79

Rent Act 1974165

Health and Safety at Work Act 197482 